\title{
Attempted Suicide, by Mail Order: Abrus precatorius
}

\author{
David H. Jang • Robert S. Hoffman • Lewis S. Nelson
}

Published online: 19 June 2010

(C) American College of Medical Toxicology 2010

\begin{abstract}
Objective Abrus precatorius is cultivated in many subtropical areas. The seeds exist in a variety of colors such as black, orange, and most commonly, glossy red. A black band is found at the end of the seed. The plant contains multiple pods which typically contain three to five Abrus seeds. The seeds contain abrin, which inhibits ribosomal function, halting protein synthesis and leading to cellular death. A unique aspect of this case is the use of the internet to order a potentially lethal poison as well as transmission of a picture to identify the seed.

Case Report A 20-year-old man presented to the emergency department complaining of vomiting and watery diarrhea for 6-8 h prior to arrival. He denied any medication use, recent illness, travel, or changes in his diet. Initial vital signs were normal. The patient was diagnosed with viral gastroenteritis. During his evaluation, the patient admitted to feeling suicidal. While awaiting psychiatry evaluation, the patient's father arrived with a box of small hard red seeds, which he believed that his son ingested in a suicide attempt. The seeds could not be identified by the staff. A picture of the seeds was transmitted by e-mail to the New York City Poison Control Center, allowing their identification as $A$. precatorius. The patient was reinterviewed and admitted to chewing and swallowing 10 seeds. Given the potential toxicity of abrin, the patient was admitted to the intensive care unit. He continued to have frequent episodes of emesis as well as diarrhea. He gradually improved over
\end{abstract}

D. H. Jang $(\varangle) \cdot$ R. S. Hoffman $\cdot$ L. S. Nelson

New York City Poison Control Center, New York University, New York, NY, USA

e-mail: Jangd01@nyumc.org

R. S. Hoffman

e-mail: bobhoffmd@gmail.com
2 days. He admitted to ordering a box of Abrus seeds online from Asia after reading on the Internet about their use in suicide. He was eventually discharged for outpatient follow-up with no permanent sequelae.

Conclusion Abrin has an estimated human fatal dose of $0.1-1 \mu \mathrm{g} / \mathrm{kg}$. Most cases of Abrus seed ingestions are unintentional and occur in children. Ingesting the intact seeds typically results in no clinical findings, as they pass through the gastrointestinal tract due to their hard shell. Abrin released during chewing is poorly absorbed systemically from the gastrointestinal tract. This causes the vomiting and diarrhea with resultant hypovolemia and electrolyte disturbances, which can be severe and life threatening, particularly in areas with less advanced health care systems. Management is primarily supportive.

Keywords Toxalbumin - Abrus precatorius $\cdot$ Abrin · Ricin . Rosary pea $\cdot$ Jequirity bean

\section{Introduction}

Abrin is a toxalbumin that can be found in the seeds of the Abrus precatorius plant. Abrin is similar in structure and function to ricin (Ricinus communis) although considered even more potent [1]. Abrin seeds, which are attractively colored and can be found in a variety of objects such as ornaments and necklaces, are most commonly ingested unintentionally by children. Occasionally, they are ingested intentionally for suicidal purposes by people who are aware of their potential toxicity [2]. The primary mechanism of abrin toxicity involves inhibition of ribosomal protein synthesis causing cellular death [9]. While the majority of patients with ingestion of Abrus seeds have good outcomes with supportive care, there are deaths reported in the literature [12]. Toxalbumins are of interest as they are 
potential biological weapons (ricin) due to their availability, potential lethality, and lack of effective antidote. A unique aspect of this case is the use of the Internet to order a potentially lethal poison as well as transmission of a picture to identify the seed.

\section{Case Report}

A 20-year-old man presented to the emergency department (ED) complaining of nausea, vomiting, and watery diarrhea for approximately $6-8 \mathrm{~h}$ prior to arrival. He denied any drug or medication use, recent illness or antibiotic use, travel, or dietary changes. He was alert and in no apparent distress. His vital signs included blood pressure of 119/ $55 \mathrm{mmHg}$, heart rate of 92 beats per minute, respiratory rate of 18 breaths per minute, and temperature of $99.8^{\circ} \mathrm{F}$. His heart and lung examinations were unremarkable. The abdomen was soft, and bowel sounds were hyperactive, and a stool sample was negative for occult blood. The general neurologic examination was normal with intact strength, sensation, cranial nerves, gait, and reflexes. Initial pertinent laboratory results included white blood cell count of 12,300 cells per cubic millimeter, hemoglobin of $11 \mathrm{~g} / \mathrm{dL}$, platelets of $390 \times 10^{3} / \mu \mathrm{L}$, and normal serum chemistry, renal function, and hepatic studies.

The patient was initially diagnosed as having viral gastroenteritis. A total of $20 \mathrm{mg}$ of metoclopramide was given for the patient's episodes of emesis and diarrhea. Two liters of intravenous normal saline was administered to correct presumed intravascular volume depletion. The patient subsequently admitted to feeling depressed but denied any pill ingestion. While awaiting psychiatric evaluation, the patient's father arrived with a box of small hard red and black seeds and he was concerned that his son ingested them in a suicide attempt (Figs. 1 and 2).

Upon the discovery of ingestion of these seeds, the New York City Poison Control Center (NYCPCC) was contacted. The seeds were described as approximately $1 \mathrm{~cm}$ in

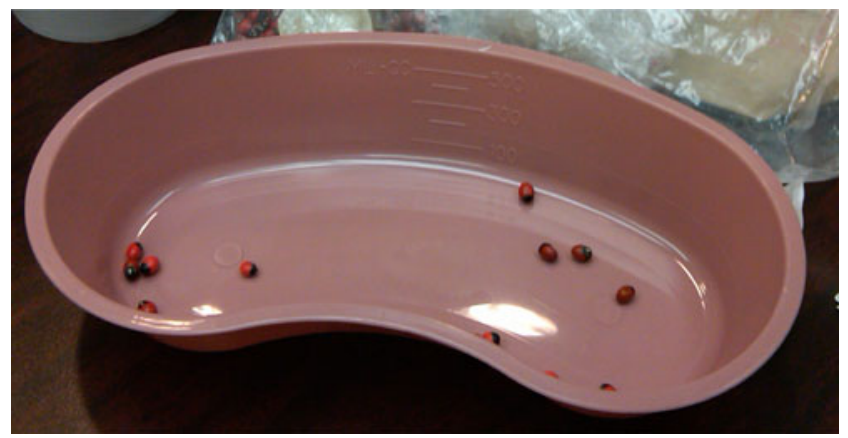

Fig. 1 The Abrus seeds brought in by family members

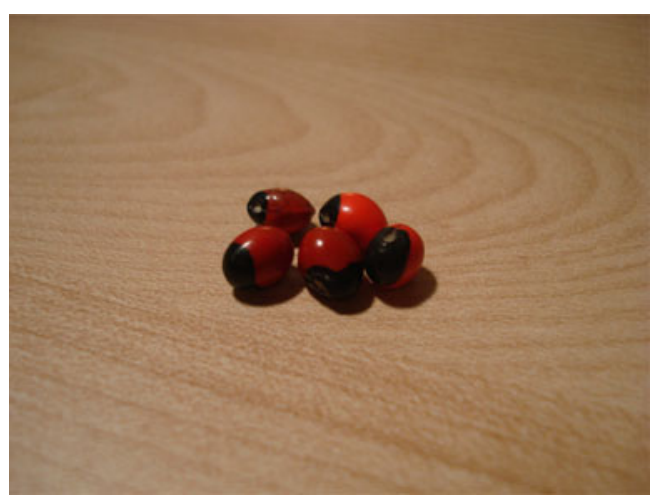

Fig. 2 Close-up of Abrus seed

size with a red shiny coat and a black band at one end. A picture of the seeds taken by a phone camera was transmitted to the NYCPCC through electronic mail, allowing their identification as $A$. precatorius. The patient was reinterviewed and admitted to chewing and swallowing 10 seeds $4-6 \mathrm{~h}$ prior to the development of his symptoms. Given the history of ingestion, an electrocardiogram was obtained that demonstrated normal sinus rhythm with normal intervals. Serum acetaminophen and salicylate concentrations were negative.

The patient was admitted to the intensive care unit (ICU) for observation and management of his fluid and electrolyte status and concern for delayed toxicity. He continued to have frequent episodes of emesis as well as diarrhea that were ameliorated with antiemetics. He developed hypokalemia to as low as $3.1 \mathrm{mEq}$, which was repleted with potassium supplementation. All his other electrolytes were normal. The patient was observed for 2 days during which time he gradually improved with correction of his electrolytes. He admitted to ordering a box of Abrus seeds online from Asia after reading on the Internet about the use of this seed in suicide. He was evaluated by psychiatry and was eventually discharged with no permanent sequelae.

\section{Discussion}

A. precatorius is a plant that originates from Southeast Asia and now can be found in subtropical areas of the world. The name Abrus, meaning beautiful or graceful, is used to describe the appearance of the seed. The seed is found in a variety of colors such as black, orange, and most commonly red with a glossy appearance with the black band at the end that attaches to the plant. The Abrus seed itself is known by a variety of names that include rosary pea, prayer bead, and jequirity bean. Precare (from which the species name is derived), meaning to pray, references its common use in rosaries [7]. 
The seeds of $A$. precatorius have been used through history in a variety of roles. Due to their uniform size and weight, they were once known as rati and used as weights for weighing gold and silver [5]. The Abrus seeds have also been used for medicinal purposes, including the treatment of chronic eye disease. Arabic culture has purportedly used the seed as an aphrodisiac known as coq's eye. Abrus has been used as a fish poison as well as a homicidal agent [7].

All parts of the plants are considered toxic. The seeds are the most toxic portion of the plant. Abrin (similar in mechanism to ricin) consists of two dissimilar, disulfidelinked polypeptide chains known as the A-chain and Bchain. The A-chain is a glycosidase that removes an adenine residue from an exposed loop of $28 \mathrm{~S}$ ribosomal RNA, which stops protein synthesis. The B-chain is the portion that binds $\beta$-D-galactopyranoside moieties on the cell membrane, allowing the complex to undergo endocytosis, bringing the A-chain internally to exert its toxic effect [8].

Abrin has an estimated human fatal dose of $0.1-1 \mu \mathrm{g} / \mathrm{kg}$, and there are reported deaths after both unintentional and intentional poisoning [7, 8]. Most cases of Abrus seed ingestion are unintentional and occur in children. Swallowing the intact seeds typically results in no clinical findings, as they pass through the gastrointestinal tract without incident due to the hard shell [10]. Abrin released during chewing is poorly absorbed systemically from the gastrointestinal tract, though the gastrointestinal mucosal cells themselves are affected. This manifests as significant vomiting and diarrhea with resultant hypovolemia and electrolyte disturbances, which can be severe and life threatening, particularly in areas with less advanced health care systems. Parenteral administration of abrin or ricin is considerably more concerning and has been associated with a high fatality rate [7]. Death in this situation is due to multisystem organ failure as cellular protein synthesis is disrupted throughout the body [9].

Patients with ingestions of chewed or otherwise damaged Abrus seeds should be admitted and observed since the onset of clinical toxicity may be delayed for many hours. While there are no antidotes for poisoning by abrin, management consists primarily of supportive care. Attention to intravascular volume status as well as electrolyte replacement should be sufficient. There are reports of rare complications associated with abrin poisoning that the clinician should be aware of such as demyelinating encephalitis as well as cerebral edema [11]. Differentiating systemic toxicity from sepsis or systemic inflammatory response syndrome can be difficult, and they may be coexistent.

There are other toxins that cause profound diarrhea such as heavy metals, colchicine, and methylxanthines, as well as certain plants. Many seeds and berries can induce gastroenteritis when ingested, either through irritation of the gastrointestinal tract (such as Dieffenbachia) or due to their content of pharmacologically active xenobiotics [6]. Pokeweed (Phytolacca americana) berries, which are green when unripe and purple when mature (and never red), induce severe gastrointestinal effects, though these berries are less toxic when mature. The presence of a mitogen in pokeweed results in a marked lymphocytosis that lasts several days [6]. The nicotine-like component found in Wisteria vine results in nicotinic cholinergic effects including vomiting and diarrhea, as well as hypertension, diaphoresis, and muscle weakness. Golden chain (Laburnum) seeds contain cytisine, and betel nut (Areca catechu) contains arecoline [6], both of which are nicotinic agonists.

There are other examples of toxalbumin-containing plants such as the castor bean ( $R$. communis) and black locust (Robinia pseudoacacia). The toxalbumin contained within the castor bean is known as ricin [6]. Ricin was first brought to the attention of the media when Georgi Markov was intentionally injected behind the knee by a hollow umbrella which quickly resulted in his death. Ricin is also a concern due to the potential to be utilized as a biological weapon. Ricin can potentially be aerosolized for inhalation which may cause widespread alveolar membrane damage rapidly leading to death. Another plant containing toxalbumin of interest is the black locust. The wood is very resistant to decomposition and extremely hard, making them useful as a fence post as well as firewood as it burns slowly with little visible smoke [6].

One of the limitations of this case is the lack of confirmatory testing. Radioimmunoassay has been used to monitor abrin and ricin concentration in cancer patients who have been treated with these toxins. This detection method is sensitive in a range of $50-100 \mathrm{pg} / \mathrm{mL}$ [3]. There is also a new method to detect exposure to abrin. This method relies on a biomarker, L-abrine, which is found as an impurity. It is excreted intact in urine which can be used as a marker of exposure to both whole and crushed seeds [4].

\section{Conclusion}

This case demonstrates minimal toxicity despite a significant reported ingestion, it is important for the clinician to realize the potential toxicity of abrin which may include seizures, cerebral edema, and death in rare cases $[9,11]$. Reported cases of severe toxicity often present with early GI symptoms but may have delayed onset of lifethreatening toxicity that would warrant ICU admission for observation [9, 11]. Severe GI toxicity and systemic involvement can occur with large ingestions of pulverized seeds as can occur through chewing. 


\section{References}

1. Dickers KJ (2003) Abrin poisoning. Toxicol Rev 22(3):137-142

2. Fernando C (2001) Poisoning due to Abrus precatorius (jequirity bean). Anaesthesia 56:1178-1180

3. Godal A, Olsnes S, Pihl A (1981) Radioimmunoassays of abrin and ricin in blood. J Toxicol Environ Health 8:409-417

4. Johnson RC, Zhou Y, Jain R et al (2009) Quantification of $\mathrm{L}$-abrine in human and rat urine: a biomarker for the toxin abrin. $\mathrm{J}$ Anal Toxicol 33:7-84

5. Nair Sankaran V (2006) The bean that weighs beads. http://www. boloji.com/environment/49.htm

6. Nelson LS, Shih RD, Balick MJ (2007) Handbook of poisonous and injurious plants. Springer/New York Botanical Garden, New York
7. Olnes S (2004) The history of ricin, abrin and related toxins. Toxicon 44:361-370

8. Olsnes S, Refsnes K, Pihl A (1974) Mechanism of action of the toxic lectins abrin and ricin. Nature 249(458):627-631

9. Palmer M, Betz JM (2006) Plants. In: Flomenbaum NE, Goldfrank LR, Hoffman RS et al (eds) Goldfrank's toxicologic emergencies, 8th edn. McGraw-Hill, New York, pp 1577-1602

10. Reedman L, Shih RD, Hung O (2008) Survival after an intentional ingestion of crushed Abrus seeds. West J Emerg Med 9(3):157159

11. Sahni V, Agarwal SK, Singh NP et al (2007) Acute demyelinating encephalitis after jequirity pea ingestion (Abrus precatorius). Clin Toxicol 45(1):77-79

12. Subrahmanyan D, Mathew J, Raj M (2008) An unusual manifestation of Abrus precatorius poisoning: a report of two cases. Clin Toxicol 46(2):173-175 